\title{
Neutrino induced transitions between the ground states of the $A=12$ triad
}

\author{
J. Engel, ${ }^{1}$ E. Kolbe, ${ }^{2}$ K. Langanke, ${ }^{3}$ and P. Vogel ${ }^{4}$ \\ ${ }^{1}$ Department of Physics, University of North Carolina, Chapel Hill, North Carolina 27599 \\ ${ }^{2}$ Departement für Physik und Astronomie, Universität Basel, Switzerland \\ ${ }^{3}$ W.K. Kellogg Radiation Laboratory, California Institute of Technology, Pasadena, California 91125 \\ ${ }^{4}$ Physics Department, California Institute of Technology, Pasadena, California 91125
}

(Received 12 June 1996)

\begin{abstract}
Neutrino induced reactions on ${ }^{12} \mathrm{C}$, an ingredient of liquid scintillators, have been studied in several experiments. We show that for currently available neutrino energies, $E_{\nu} \leqslant 300 \mathrm{MeV}$, calculated exclusive cross sections ${ }^{12} \mathrm{C}_{\text {g.s. }}(\nu, l){ }^{12} \mathrm{~N}_{\text {g.s. }}$ for both muon and electron neutrinos are essentially model independent, provided the calculations simultaneously describe the rates of several other reactions involving the same states or their isobar analogs. The calculations agree well with the measured cross sections, which can be therefore used to check the normalization of the incident neutrino spectrum and the efficiency of the detector. [S0556-2813(96)01011-4]
\end{abstract}

PACS number(s): 13.15. $+\mathrm{g}, 21.60 .-\mathrm{n}, 23.40 . \mathrm{Bw}, 25.30 . \mathrm{Pt}$

A solid theoretical understanding of cross sections in neutrino induced reactions on light nuclei is becoming a necessity, in particular for ${ }^{12} \mathrm{C}$, an ingredient of liquid scintillators, and for ${ }^{16} \mathrm{O}$, the basic component of water Cerenkov detectors. Detectors containing these isotopes are used to measure fluxes of atmospheric and supernova neutrinos, and in searches for neutrino oscillations. These tasks to a large extent require knowledge of the corresponding cross sections, which often have not been measured. When experimental cross sections are available it is therefore important to compare them with calculations.

For ${ }^{12} \mathrm{C}$ a number of experimental results exist. These include measurements of charged-current reactions induced by both electron, [1] and muon neutrinos [2-4], exciting both the ground and continuum states in ${ }^{12} \mathrm{~N}$. The inclusive cross section for ${ }^{12} \mathrm{C}\left(\nu_{e}, e\right){ }^{12} \mathrm{~N}^{*}$, measured by Karmen [1], LAMPF (with large errors) [5], and recently by the LSND Collaboration [4], agree well with calculations. (A previously reported measurement of both exclusive and inclusive neutrino cross sections on ${ }^{12} \mathrm{C}$ [2] appears to be inconsistently large [3]; we will not consider that measurement here.) By contrast, there is a disturbing discrepancy between calculations [6-8] and the LSND value of the cross section for the inclusive reaction ${ }^{12} \mathrm{C}\left(\nu_{\mu}, \mu\right){ }^{12} \mathrm{~N}^{*}$, which uses higher energy neutrinos from pion decay in flight $[3,4,9]$. The disagreement is disturbing ${ }^{1}$ in light of the apparent simplicity of the reaction and in view of the fact that parameter-free calculations, such as those in $[6,7]$ describe well other weak processes which are governed by the same weak current nuclear matrix elements. Here we examine whether similar problems affect our understanding of the exclusive reactions ${ }^{12} \mathrm{C}\left(\nu_{e}, e\right){ }^{12} \mathrm{~N}_{\text {g.s. }}[1,3]$ and ${ }^{12} \mathrm{C}\left(\nu_{\mu}, \mu\right){ }^{12} \mathrm{~N}_{\text {g.s. }}[3,4,9]$. The exclusive process is a useful monitor of the neutrino flux. If calculations are reliable and reproduce experiment, then the

\footnotetext{
${ }^{1}$ The disagreement is not universal. In [10] a cross section that agrees with LSND is obtained using the elementary particle method. We discuss the applicability of the approximations used in [10] elsewhere [11].
}

normalization of the experimental neutrino flux must have been correctly modeled.

A calculation of the exclusive cross section can be tested by computing rates of related processes and comparing to data. The $J^{\pi}, T=1^{+}, 1$ ground state in ${ }^{12} \mathrm{~N}$ is the analog of the $15.11 \mathrm{MeV}$ state in ${ }^{12} \mathrm{C}$ and of the ground state of ${ }^{12} \mathrm{~B}$. This allows us to use $\beta^{+}$decay from ${ }^{12} \mathrm{~N}$ back to the $J^{\pi}, T=0^{+}, 0$ ground state of ${ }^{12} \mathrm{C}$, muon capture from ${ }^{12} \mathrm{C}$ to the ground state of ${ }^{12} \mathrm{~B}$, ineleastic electron scattering to the $15.11 \mathrm{MeV}$ state in ${ }^{12} \mathrm{C}$, and $M 1$ decay from that state to the ground state to calibrate elements of the calculation. In what follows we calculate the exclusive neutrino cross sections in several ways to see whether when all the above data are reproduced, the different models can produce significantly different predictions.

Our first approach is a restricted shell-model calculation. Describing all the above reactions is straightforward in the shell model as long as we can neglect contributions of twobody operators (i.e., meson exchange). The reduced matrix element of an arbitrary operator $\hat{O}$ is then given by

$$
\left\langle 1^{+}, 1\|\hat{O}\| 0^{+}, 0\right\rangle=\sum_{j, j^{\prime}}\left\langle j\|\hat{O}\| j^{\prime}\right\rangle \mathrm{OBD}\left(j, j^{\prime}\right),
$$

where the one-body transition densities (OBD's) are defined by

$$
\operatorname{OBD}\left(j, j^{\prime}\right)=\left\langle 1^{+}, 1\left\|\left[a_{j}^{\dagger} \overrightarrow{a_{j}}\right]^{J^{\pi}, T=1^{+}, 1}\right\| 0^{+}, 0\right\rangle .
$$

If we further assume that all of the structure in the low-lying states with $J^{\pi}, T=1^{+}, 1$ and $0^{+}, 0$ is generated by the eight "valence" nucleons moving in the $p$ shell, there are only four one-body densities and four single-particle matrix elements $\left\langle j\|\hat{O}\| j^{\prime}\right\rangle$, which contain all of the momentumtransfer dependence and which are simple to calculate. Futhermore, the Gamow-Teller (GT) matrix element for the $\beta^{+}$ decay of ${ }^{12} \mathrm{~N}$, the $M 1$ radiative width of the state at 15.11 $\mathrm{MeV}$ in ${ }^{12} \mathrm{C}$, and the form factor for the excitation of this state in electron scattering [12] depend only on three one- 
TABLE I. Fitted one-body densities $\operatorname{OBD}\left(j, j^{\prime}\right)$. The parameter $b$ is the oscillator length. The range in line 2 , column 4 , reflects the uncertainty in the muon capture rate; in other lines only the most probable capture rate was used.

\begin{tabular}{cccccc}
\hline \hline & & & & $b$ \\
$j, j^{\prime}$ & $1 / 2,1 / 2$ & $1 / 2,3 / 2-3 / 2,1 / 2$ & $1 / 2,3 / 2+3 / 2,1 / 2$ & $3 / 2,3 / 2$ & $(\mathrm{fm})$ \\
\hline Present & -0.113 & 0.106 & $0.666 \pm 0.4$ & 0.24 & 1.67 \\
Ref. [15] & -0.111 & 0.337 & 0.875 & 0.086 & 1.76 \\
Ref. [16] & 0.0 & 1.0 & 1.0 & 0.0 & 1.77 \\
Ref. [17] & -0.27 & 0.318 & 1.03 & 0.116 & 1.82 \\
\hline \hline
\end{tabular}

body densities; they are independent of the combination $\operatorname{OBD}\left(p_{3 / 2}, p_{1 / 2}\right)+\operatorname{OBD}\left(p_{1 / 2}, p_{3 / 2}\right)$. (See the review [13] for a general discussion.)

The most straightforward way of obtaining the one-body densities is by diagonalizing a thoroughly tested residual interaction, such as the one given in Ref. [14]. It is well known, however, that the resulting $p$-shell one-body densities do not describe the reactions above very well. Typically, the calculated GT strength is too large (the origin of "GT quenching") and the electron scattering form factor is too high in the first lobe. In a number of papers [15-18] over the last 20 plus years, however, it has been shown that one can modify the one-body densities (ad hoc) in such a way that the three experiments are correctly reproduced, with the form factor adequately described up to the first minimum at $|\vec{q}| \approx 1.5 \mathrm{fm}^{-1}$. This is the approach we follow here, adjusting the densities to reproduce the data. The so-far undetermined fourth combination of densities can be fixed (with some uncertainty) by the muon capture rate to the ground state of ${ }^{12} \mathrm{~B}$ [13]. This process is the only one that tests the momentum dependence of the axial current, since it takes place at $q^{2}=-0.74 m_{\mu}^{2}$. For our analysis we take an averaged experimental rate $\omega\left(1^{+}\right)=6200 \pm 200 \mathrm{~s}^{-1}$ [19] and use the Goldberger-Treiman relation for the inducedpseudoscalar coupling constant.

Line 2 of Table I contains the resulting one-body densities, adjusted here to reproduce all the data discussed above. They are chosen in such a way that no further adjustment of form factors (in particular of $F_{A}$ and $F_{M}$ ) is needed. In contrast, lines 3-5 contain one-body densities used by other authors, and constrained to different subsets of the above data. To achieve an overall agreement with all the data, we renormalize, in addition, either the weak axial form factor $F_{A}$ or the magnetic form factor $F_{M}$. Haxton [15] (line 3) required that the $\beta^{-}$decay of ${ }^{12} \mathrm{~B}$, rather than ${ }^{12} \mathrm{~N}$ (but not the muon capture), be well described; because of isospin violation in the $f t$ values, his densities require a renormalization factor of 0.873 for the axial current form factor $F_{A}$ to fit the ${ }^{12} \mathrm{~N}$ decay. The entries in line 4 are based on the extreme singleparticle model [16], in which ${ }^{12} \mathrm{C}$ is represented as a closed $p_{3 / 2}$ subshell, and ${ }^{12} \mathrm{~N}$ or ${ }^{12} \mathrm{~B}$ have one nucleon in $p_{1 / 2}$ subshell. Here a renormalization of 0.414 is required for $F_{A}$, and 0.484 for the magnetic form factor $F_{M}$. Finally, line 5 contains the one-body densities of Ref. [17], based on a minimally modified Cohen-Kurath interaction. They require only a small $F_{A}$ renormalization of 0.925 . In all cases harmonic oscillator wave functions are used, with slightly different values of the oscillator parameter $b$ taken from the original references. The table clearly illustrates that the extraction of one-body densities from data is not a unique procedure; it depends on other assumptions about wave functions, etc. We discuss the effects of these differences on the exclusive neutrino cross sections shortly.

While two-body effects, i.e., meson exchange corrections, are expected to be relatively small $(5-10 \%)$ for the momentum transfer considered here [20], configurations beyond the $p$ shell might explain the need for a drastic renormalization of the one-body densities produced by a reasonable $p$-shell Hamiltonian. We therefore calculate the rates of all the reactions above, including exclusive neutrino capture, in the random phase approximation (RPA), which does include multishell correlations, while treating the configuration mixing within the $p$ shell only crudely as sketched below. The same method, extended to continuum, has been used in the calculation of the neutrino charged and neutral current inclusive scattering $[6,7,21]$ (disagreeing disturbingly with one experiment, as noted above) and muon capture [22].

Only certain configurations of the eight $p$ nucleons are present in the RPA. The ${ }^{12} \mathrm{C}$ ground state is represented by the occupied $p_{3 / 2}$ and empty $p_{1 / 2}$ subshells with the ground state correlation admixtures of two-particle-two-hole, fourparticle-four-hole, etc., states determined by the RPA equations of motion, not by the complete diagonalization as in the shell model. Similarly, the ground state of ${ }^{12} \mathrm{~N}$ is represented by a one- $p_{3 / 2}$-hole-one- $p_{1 / 2}$-particle state with admixtures of three-particle-three-hole, etc., states again determined by the same RPA equation of motion as the ${ }^{12} \mathrm{C}$ ground state. To compensate for this somewhat crude description of the $p$-shell dynamics we use an overall quenching factor of 0.258 by which we multiply the rates, respectively, the cross sections of all processes under consideration, for all momentum transfers. With this multiplicative factor the $\beta^{+}$decay, $M 1$ width, electron scattering form factor, and partial muon capture rate are all adequately described, and the exclusive cross sections, discussed below, are readily calculated. (It has been known for some time that the RPA is capable of describing the shape of the $\left(e, e^{\prime}\right)$ form factor for the 15.11 $\mathrm{MeV}$ state [23].)

Our third approach is the "elementary-particle treatment" (EPT). Instead of describing nuclei in terms of nucleons or other constituents, the EPT considers them elementary and describes transition matrix elements in terms of nuclear form factors deduced from experimental data and constrained by transformation properties. The EPT was implemented in the $A=12$ system in Refs. [24-26] for neutrino energies up to $E_{\nu}=100 \mathrm{MeV}$. Here we extend the approach to the higher neutrino energies relevant to the LSND decay in flight $\nu_{\mu}$ 's by appropriately including the lepton mass, which was ignored in Ref. [24], in the kinematics. A nonzero lepton mass requires, in turn, that the induced-pseudoscalar term, also neglected in Ref. [24], must be included as well. Here we used a modified form of Eq. (16) in Ref. [24], kindly furnished to us by Professor Kubodera and for the $F_{P}\left(Q^{2}\right)$ we used the simple ansatz

$$
F_{P}\left(Q^{2}\right)=-\frac{m_{\pi}^{2}}{Q^{2}} F_{A}(0)\left[F_{A}\left(Q^{2}\right)-\frac{m_{\pi}^{2}}{Q^{2}+m_{\pi}^{2}} \frac{1}{1+Q^{2} / \xi^{2}}\right],
$$


TABLE II. Comparison of calculated and measured cross sections, in units of $10^{-42} \mathrm{~cm}^{-2}$ and averaged over the corresponding neutrino spectra, for the neutrino induced transitions ${ }^{12} \mathrm{C}_{\text {g.s. }} \rightarrow{ }^{12} \mathrm{~N}_{\text {g.s. }}$ and ${ }^{12} \mathrm{C}_{\mathrm{g} . s .} \rightarrow{ }^{12} \mathrm{~B}_{\text {g.s. }}$. For the decay at rest the $\nu_{e}$ spectrum is normalized from $E_{\nu}=0$ while for the decay in flight the $\nu_{\mu}$ and $\bar{\nu}_{\mu}$ spectra are normalized from the corresponding threshold. The cross section for $\bar{\nu}_{e}$ is not quoted since the decay-at-rest neutrino source does not contain any $\bar{\nu}_{e}$ 's.

\begin{tabular}{lccc}
\hline \hline & $\begin{array}{c}{ }^{12} \mathrm{C}\left(\nu_{e}, e^{-}\right)^{12} \mathrm{~N}_{\text {g.s. }} \\
\text { Decay at rest }\end{array}$ & $\begin{array}{c}{ }^{12} \mathrm{C}\left(\nu_{\mu}, \mu^{-}\right){ }^{12} \mathrm{~N}_{\mathrm{g} . s .} \\
\text { Decay in flight }\end{array}$ & $\begin{array}{c}{ }^{12} \mathrm{C}\left(\bar{\nu}_{\mu}, \mu^{+}\right)^{12} \mathrm{~B}_{\text {g.s. }} \\
\text { Decay in flight }\end{array}$ \\
\hline Experiment & $8.9 \pm 0.6 \pm 0.75[1]$ & - & - \\
Experiment & $9.1 \pm 0.4 \pm 0.9[4]$ & $64 \pm 10 \pm 10[4]$ & - \\
Experiment & $10.5 \pm 1.0 \pm 1.0[5]$ & - & - \\
OBD of this work & $9.1^{\mathrm{a}}$ & $63.5^{\mathrm{b}} \pm 5$ & 24 \\
OBD of Ref. [15] & 8.8 & 60.4 & 23 \\
OBD of Ref. [16] & 9.4 & 65.4 & 23.6 \\
OBD of Ref [17] & 9.4 & 62.3 & 26 \\
RPA [6,7] & 9.3 & 63.0 & $21.5^{\mathrm{c}}$ \\
EPT [24] & 9.2 & $62.9^{\mathrm{c}}$ & \\
\hline
\end{tabular}

${ }^{a}$ The uncertainty in the muon capture rate causes only a very small uncertainty of \pm 0.1 in this cross section. ${ }^{\mathrm{b}}$ The uncertainty reflects the range corresponding to the uncertainty in the muon capture rate. A similar range is presumably valid for the other approaches in lines 4-6.

${ }^{\mathrm{c}}$ Extended to muon neutrinos, see text.

where, as usual, $Q^{2}=-q^{2}$, and the empirical parameter $\xi$ has been fixed from muon capture.

We turn now to the evaluation of the exclusive cross sections in the three approaches. Within the shell model and the RPA the cross section is easily evaluated once the one-body densities and free-nucleon form factors (for which we use standard values) are specified; the general prescription can be found, e.g., in Ref. [27]. In the EPT the evaluation is even simpler since the nucleus is elementary. One complication often not considered is related to the Coulomb interaction of the outgoing charged lepton. The usual treatment, as in nuclear $\beta$ decay, involves the Fermi function $F(Z, E)$, which is the ratio of the Coulomb continuum $s$ wave and the corresponding free $s$-wave wave. This approximation is valid, however, only for lepton momenta $p R \leqslant 1$, where $R$ is the nuclear radius. For ${ }^{12} \mathrm{C}$ it can be used for electrons with up to

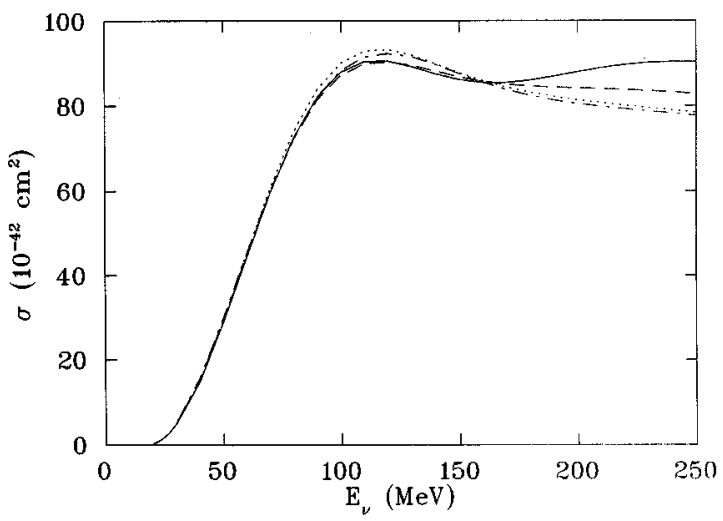

FIG. 1. Cross section for ${ }^{12} \mathrm{C}\left(\nu_{e}, e\right){ }^{12} \mathrm{~N}_{\text {g.s. }}$ as a function of the $\nu_{e}$ energy, in units of $10^{-42} \mathrm{~cm}^{2}$. The full line is for the shell model with one-body densities from line 2, Table I, the dashed line is the modified Cohen-Kurath one-body densities from line 5, Table I [17] (the curves for the other shell-model variants are very similar and are not shown), the dot-dashed line is for the RPA [7], and the dotted line is for the EPT. about $50 \mathrm{MeV}$ of kinetic energy, but it is justified only for muons of $10 \mathrm{MeV}$ or less of kinetic energy. As energy increases, $F(Z, E)$ approaches a constant value, $\sim 1.17$ $(Z=7)$ for $\nu$ reaction on ${ }^{12} \mathrm{C}$, and $\sim 0.90(Z=5)$ for $\bar{\nu}$ on ${ }^{12} \mathrm{C}$. On the other hand, it is intuitively clear that as the lepton energy becomes much larger than the Coulomb energy, the Coulomb correction should approach unity. In order to keep things simple, and since the correction is a relatively minor one, we scale at higher energies the lepton cross section by the ratio

$$
\frac{p_{\mathrm{eff}}(E+\langle V\rangle)}{p E}
$$

where

$$
p_{\text {eff }}=p\left(1+\frac{\langle V\rangle}{E}\right), \quad\langle V\rangle= \pm \frac{3 Z \alpha}{2 R}
$$

and $\langle V\rangle$ represents the average Coulomb potential. The two approximations are smoothly connected.

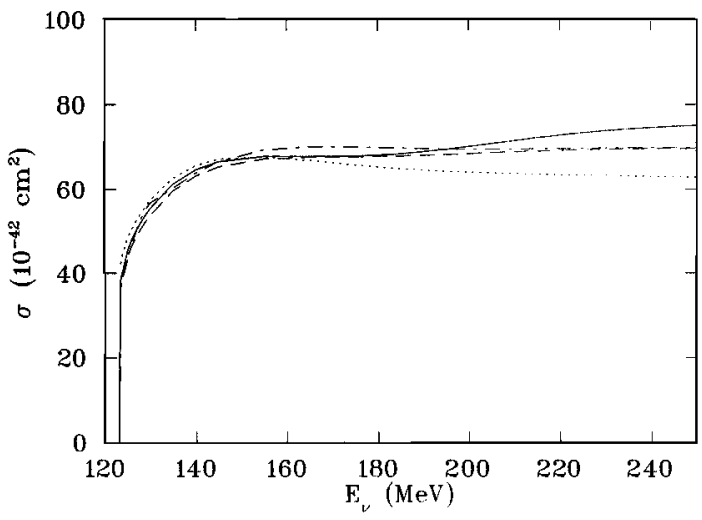

FIG. 2. Cross section for ${ }^{12} \mathrm{C}\left(\nu_{\mu}, \mu\right){ }^{12} \mathrm{~N}_{\text {g.s. }}$ as a function of the $\nu_{\mu}$ energy, in units of $10^{-42} \mathrm{~cm}^{2}$. The notation is as in Fig. 1. 
TABLE III. Comparison of calculated and measured cross sections, in units of $10^{-42} \mathrm{~cm}^{-2}$ and averaged over the corresponding neutrino spectra, for the neutrino-induced neutral-current transitions ${ }^{12} \mathrm{C}_{\text {g.s. }}\left(\nu, \nu^{\prime}\right){ }^{12} \mathrm{C}(15.11)$. In column 2 the contributions from $\nu_{e}$ and $\bar{\nu}_{\mu}$ are added. All spectra are normalized "per neutrino,", i.e., from $E_{\nu}=0$.

\begin{tabular}{lccc}
\hline \hline & $\begin{array}{c}{ }^{12} \mathrm{C}\left(\nu, \nu^{\prime}\right){ }^{12} \mathrm{C}(15.11) \\
\text { Decay at rest, } \nu:=\nu_{e}+\bar{\nu}_{\mu}\end{array}$ & $\begin{array}{c}{ }^{12} \mathrm{C}\left(\nu_{\mu}, \nu_{\mu}^{\prime}\right){ }^{12} \mathrm{C}(15.11) \\
\text { Decay in flight }\end{array}$ & $\begin{array}{c}{ }^{12} \mathrm{C}\left(\bar{\nu}_{\mu}, \vec{\nu}_{\mu}\right){ }^{12} \mathrm{C}(15.11) \\
\text { Decay in flight }\end{array}$ \\
\hline Experiment & $11 \pm 1.0 \pm 0.9[1]$ & - & - \\
OBD of this work & 9.8 & 23.5 & 16.0 \\
OBD of Ref. [15] & 9.8 & 23.0 & 16.1 \\
OBD of Ref. [16] & 9.9 & 24.9 & 17.3 \\
OBD of Ref [17] & 10.1 & 24.1 & 16.7 \\
RPA [6,7] & 10.5 & 27.2 & 17.5 \\
EPT [24] & 9.9 & 25.6 & 15.3 \\
\hline \hline
\end{tabular}

The results appear in Table II. For the $\nu_{e}$-induced reaction with neutrinos from the muon decay at rest [1] the agreement between the experimental and calculated exclusive cross section is perfect in all the models. This cross section, corresponding to an average momentum transfer of only about 50 $\mathrm{MeV}$, appears totally fixed by the requirements we impose on each of the calculations. For the $\nu_{\mu}$-induced reaction the average momentum transfer is about $150 \mathrm{MeV}$. But even in this case the different variants of the shell model, the RPA and the EPT give quite similar cross sections. Again, the agreement with the LSND experimental value is good.

In Figs. 1 and 2 we show the exclusive cross sections for ${ }^{12} \mathrm{C}\left(\nu_{e}, e\right){ }^{12} \mathrm{~N}_{\text {g.s. }}$ and ${ }^{12} \mathrm{C}\left(\nu_{\mu}, \mu\right){ }^{12} \mathrm{~N}_{\text {g.s. }}$, respectively, as a function of neutrino energy. For $\nu_{e}$ 's the cross section is essentially the same in all the approaches we consider. The agreement between the various models is also quite good for $\nu_{\mu}$ 's. Moreover, after a very steep rise from the threshold, this cross section quickly saturates and becomes more or less independent on the neutrino energy. Thus, the exclusive cross section is simply proportional to the total number of $\nu_{\mu}$ 's above threshold. However, it is essentially independent of their energy distribution.

For completeness, Table III lists the cross sections for neutral-current excitation of the $15.11 \mathrm{MeV}, J^{\pi}, T=1^{+}, 1$ state in ${ }^{12} \mathrm{C}$, which was measured by Karmen [1] for neutri- nos from decay at rest. Once again all the models agree with one another and the data. For the monoenergetic $\nu_{\mu}$ from pion decay at rest the calculated neutral current cross section is $2.6 \times 10^{-42} \mathrm{~cm}^{2}$ for the OBD of this work. The neutralcurrent cross section for $\nu_{\mu}$ 's from decay-in-flight and for $\bar{\nu}_{\mu}$ 's are probably not measurable, but unsurprisingly the calculations continue to agree with one another.

In conclusion, we have shown that the exclusive cross sections for neutrino energies available now are calculable in a variety of ways, with results that are nearly identical, and agree very well with the data. These processes can therefore be used as a check of the neutrino flux normalization, and detector efficiency and indicate that the discrepancy between the measured and calculated inclusive ${ }^{12} \mathrm{C}\left(\nu_{\mu}, \mu\right){ }^{12} \mathrm{~N} *$ cross sections is not caused by an underestimate of the neutrino flux normalization. The source of the disagreement must be found elsewhere.

We would like to thank Professor Kubodera for his help with the EPT. We also appreciate numerous discussions with the members of LSND Collaboration. We were supported in part by the U.S. Department of Energy under Grants DEFG05-94ER40827 and DE-FG03-88ER-40397, by the U.S. National Science Foundation under Grants PHY94-12818 and PHY94-20470, and by the Swiss National Science Foundation.
[1] G. Drexlin et al., KARMEN Collaboration, in Proceedings of the Neutrino Workshop, Heidelberg, 1987, edited by B. Povh and V. Klapdor (Springer-Verlag, Berlin, 1987), p. 147; G. Drexlin et al., KARMEN Collaboration, Phys. Lett. B 267, 321 (1991); B. Zeitnitz, KARMEN Collaboration, Prog. Part. Nucl. Phys. 32, 351 (1994).

[2] D.D. Koetke et al., Phys. Rev. C 46, 2554 (1992).

[3] M. Albert et al., Phys. Rev. C 51, 1065 (1995).

[4] H. J. Kim et al., unpublished.

[5] D.A. Krakauer et al., Phys. Rev. C 45, 2450 (1992).

[6] E. Kolbe, K. Langanke, and S. Krewald, Phys. Rev. C 49, 1122 (1994).

[7] E. Kolbe, K. Langanke, F.-K. Thielemann, and P. Vogel, Phys. Rev. C 52, 3437 (1995).
[8] T. S. Kosmas and E. Oset, Phys. Rev. C 53, 1409 (1996).

[9] C. Athanassopoulos, Ph.D. thesis, Temple University, 1995, unpublished.

[10] S. L. Mintz and M. Pourkaviani, Nucl. Phys. A594, 346 (1995).

[11] E. Kolbe, K. Langanke, and P. Vogel, submitted to Nucl. Phys. A.

[12] U. Deutschmann, G. Lahm, R. Neuhausen, and J. C. Bergstrom, Nucl. Phys. A411, 337 (1983); R. S. Hicks et al., Phys. Rev. C 36, 485 (1987).

[13] T. W. Donnelly and R. D. Peccei, Phys. Rep. 50, 1 (1979).

[14] E. K. Warburton and B. A. Brown, Phys. Rev. C 46, 923 (1992).

[15] W. C. Haxton, Phys. Lett. 76B, 165 (1978). 
[16] J. S. O'Connell, T. W. Donnelly, and J. D. Walecka, Phys. Rev. C 6, 719 (1972).

[17] F. P. Brady et al., Phys. Rev. C 43, 2284 (1991).

[18] B. C. Doyle and N. C. Mukhopadhyay, Phys. Rev. C 52, 1947 (1995).

[19] Y. G. Budyashov et al., Sov. Phys. JETP 31, 651 (1970); G. H. Miller et al., Phys. Lett. 41B, 50 (1972); L. Ph. Roesch et al., ibid. 107B, 31 (1981).

[20] Y. Umino and J. M. Udias, Report nucl-th 9602003.

[21] E. Kolbe, K. Langanke, S. Krewald, and F.-K. Thielemann, Nucl. Phys. A540, 599 (1992).
[22] E. Kolbe, K. Langanke, and P. Vogel, Phys. Rev. C 50, 2576 (1994).

[23] T. Suzuki, F. Osterfeld, and J. Speth, Phys. Lett. 100B, 443 (1981).

[24] M. Fukugita, Y. Kohyama, and K. Kubodera, Phys. Lett. B 212, 139 (1988).

[25] K. Kubodera and S. Nozawa, Int. J. Mod. Phys. 3, 101 (1994).

[26] S. Nozawa, Y. Kohyama, and K. Kubodera, Prog. Theor. Phys. 70, 892 (1983).

[27] J. D. Walecka, in Muon Physics, edited by V. W. Hughes and C. S. Wu (Academic Press, New York, 1977), Vol. II, p. 113. 